\title{
\#México19s: transformación digital ante la emergencia
}

\author{
Igor Jiménez y Emiliano Valdés
}

\section{Resumen}

En el contexto del sismo del 19 de septiembre de 2017 las plataformas digitales tuvieron un papel primordial para compartir la información y facilitar la ayuda. Sin embargo, dichas plataformas también se prestaron a la desinformación. En este artículo se reflexiona acerca de la experiencia de uso de las herramientas y medios digitales en dichas circunstancias, para comprender el momento evolutivo en el que estábamos y en el que nos encontramos actualmente con relación a la transformación y cultura digital.

El presente documento se basa en la experiencia de colaboración entre coDeck (un equipo interdisciplinario que integra la ciencia de datos, el análisis social y la interacción digital en consultorías) y el Huerto Roma Verde (una comunidad activa involucrada en el bienestar común de su entorno que realiza proyectos socio-ambientales). En los álgidos días que devinieron al terremoto se implementó una propuesta, sustentada en la inteligencia artificial, que abarcaba una visión accionable y de herramienta. Precisamente la fricción entre la idea y la implementación en un contexto completamente único, derivado del estado de emergencia, generaron dinámicas dialécticas que se analizan con el fin de compartir el aprendizaje. No sobra decir que todo esto fue posible en un contexto de emergencia donde internet y la electricidad seguían funcionando, y que mucho de lo expuesto aquí no tendría utilidad si el desastre fuera de mayor magnitud.

Palabras clave: sismo, 19s, innovación social, ciudadanía, resiliencia, crisis, empatía, ayuda, cultura digital, transformación digital, modelos matemáticos, inventarios, bases de datos, redes sociales, algoritmos. 


\title{
\#MEXICO19s: DIGITAL TRANSFORMATION BEFORE THE EMERGENCY
}

\begin{abstract}
During the Mexico City earthquake in September 19th, digital platforms had a primordial role in sharing information and facilitating help. However, those platforms also contributed to disinformation. In this article we reflect about the use of digital media and digital tools in such circumstances in order to understand the evolutionary moment in which we were then and the one in which we are now in the context of digital transformation and digital culture.

This text is based in the collaboration experience between coDeck (an interdisciplinary team that integrates data science, social analysis and digital interaction into consultancies) and Huerto Roma Verde (an active community involved in common welfare that coordinates socioenvironmental projects). A proposal was implemented in those crucial days after the earthquake; it was based on artificial intelligence with the intention of being actionable as well as a useful tool. The friction between ideas and its implementation in this unique context, consequence of the state of emergency, created dialectical dynamics that are analyzed with the objective of sharing what we learned from them. It is important to stress that all of this was possible in an emergency context where Internet connection and electricity were still working, and that much of what we propose would not have been useful if this natural disaster had been of greater magnitude.
\end{abstract}

Keywords: earthquake, September 19th, social innovation, citizenship, resilience, crisis, empathy, help, digital culture, digital transformation, mathematical models, inventories, database, social media, algorithms. 


\section{Emiliano Valdés Guerrero}

\section{emiliano@co-deck.com}

Es matemático de la Facultad de Ciencias de UNAM. Socio fundador y director de tecnología (CTO) en CoDeck, una empresa emergente de Innovación y Ciencia de Datos. A pesar de haber estudiado matemáticas puras su pasión son las matemáticas aplicadas, los modelos y algoritmos que permiten resolver problemas reales. Ha desarrollado su labor profesional en modelación matemática, ciencia de datos, big data, diseño de software, inteligencia artificial, aprendizaje automatizado y agentes autónomos. Es también un apasionado de la docencia y la divulgación de las matemáticas, ha participado en distintos foros y en una amplia gama de cursos para estos fines desde el 2004.

\section{Igor Jiménez}

igor@co-deck.com

Graduado del Instituto Superior de arte de La Habana, Cuba en los años noventa, dedicó sus primeros años a la exploración del lenguaje audiovisual realizando desde video poemas, ejecuciones de visuales en vivo con música, documentales confrontativos, exploraciones en ficción que incluían la improvisación o narrativas no-lineales en cortometrajes mostrados en cuatro continentes, obteniendo algunos premios fuera de México.

Durante un periodo vivió en España y comenzó una carrera comercial en publicidad misma que lo llevó a dirigir spots de publicidad en España, Argentina, Venezuela, Chile, México y Estados Unidos. Su camino de exploración personal del lenguaje audiovisual, lo llevó a generar una plataforma de video interactivo a principio de los 2000, que más tarde se convertiría en una aplicación de patente en el Reino Unido. A partir de ese momento su camino hacia la interactividad no tendría retorno y comenzaría a adentrarse en el estudio de esta disciplina para terminar siendo uno de los líderes locales en CDMX de la Interactive Design Foundation, al obtener varias certificaciones con esta institución. En 2017, junto a Emiliano Valdés y Emmanuel Flores, funda CoDeck con la misión de introducir en México y Latinoamérica, una cultura basada en la ciencia de datos, que permita a las empresas generar estrategias financieras, comerciales y de marketing, e innovaciones de productos y servicios con un diseño centrado en las personas. En este nuevo recorrido, CoDeck ha co-creado desde la inmadurez digital del mercado mexicano y el potencial todavía incipiente para impulsarlo hacia una verdadera transformación digital. 


\section{Introducción}

En la 32 conmemoración del terremoto de 1985 nos llegó el recordatorio físico de nuestra condición geográfica, con el pánico en nuestros sistemas nerviosos, comenzamos a conocer las consecuencias de la tragedia y, sobre todo, a comunicarnos; la comunicación verbal no hacía más que precipitarnos a la recepción y emisión de más mensajes digitales. Durante aquellos días, la intersección de estos dos fenómenos, lo humano y lo digital, trabajando como un nuevo ente de la evolución humana, nos dejó muchas cosas para reflexionar y aprender, sobre todo si no olvidamos que lo único seguro para nosotros, los habitantes de la Ciudad de México (CDMX), es que tendremos más terremotos.

La ciudadanía que estaba cerca de los derrumbes originaba cadenas de información valiosa que se replicaba aceleradamente por los medios digitales, re-convirtiéndose en oportunidades para la solidaridad y amplificando el impacto del suceso natural. Debemos visualizar que nos desenvolvemos en un contexto de ingesta de información acelerada, a la que nos sometemos diariamente por voluntad propia, donde se expresa nuestra incultura digital (en tanto proceso inédito en nuestra evolución).

En aquellas horas vivimos bajo los efectos de un cóctel bioquímico extraordinario en nuestros sistemas nerviosos (Charney, 2004: 195) y eso, aunado a la impulsividad y la impotencia del momento, terminó por provocar lo que podríamos llamar patrones de uso indiscriminado de los medios digitales, es decir: todas aquellas cadenas de reenvíos de información que se hacían compulsivamente al calor de esa agitación bioquímica producida por el trauma colectivo y que eran amplificadas en plataformas como WhatsApp, Twitter o Facebook.

En aquellas cadenas, la veracidad se diluía en un trasiego de información que venía de todos lados y que, a fuerza de ser replicadas fuera de contexto, generaban desinformación. Algunas de ellas nacieron con una vocación de posverdad o fake news (Coughlan, 2017), incluso adjudicándose la voz de instituciones oficiales. No obstante, la mayoría eran intentos de colaboración que satisfacían la sensación de pertenencia a la que nos veíamos impulsados desde el estrés y el trauma, el fenómeno se potenciaba con la necesidad de empatía, para terminar por erigirse en una realidad alterna, una suerte de histeria digital, que se mezclaba con ese espacio otro de vida, aquel que nuestros cerebros, desde un punto de vista evolutivo, sólo comienzan a conocer desde hace pocos años y que no es otro sino el espacio digital.

Esta incontinencia digital que nos sobrevino, entró al cabo de los días en un proceso de autorregulación, atribuible en parte a un distanciamiento postraumático, a la consecuente dilución de sustancias bioquímicas producidas por el trauma en los habitantes de la Ciudad, y a la aparición de mensajes digitales que solicitaban la confirmación de la información antes de su reproducción. 
1 "La resiliencia es el proceso de adaptarse bien a la adversidad, a un trauma, tragedia, amenaza, o fuentes de tensión significativas [...].

Significa «rebotar» de una experiencia difícil, como si uno fuera una bola o un resorte [...] Ser resiliente no quiere decir que la persona no experimenta dificultades o angustias [...] De hecho, el camino hacia la resiliencia probablemente está lleno de obstáculos que afectan nuestro estado emocional" (Asociación Americana de Psicología, El Camino a la Resiliencia).

2 La gobernanza "promueve la equidad, la participación, el pluralismo, la transparencia, la responsabilidad y el estado de derecho, de modo que sea efectivo, eficiente y duradero" (Naciones Unidas).

3 La transformación digital impulsa nuevos modelos de negocio basados en análisis de big data, procesos de machine learning e inteligencia artificial, generalizando podemos decir que inciden en la eficiencia y la transparencia; mientras que la cultura digital es el conjunto de acciones que utilizan ese espacio en sus diversas facetas para consolidar los procesos que puedan derivar en una transformación digital.

\section{Resiliencia e incultura digital}

En los días posteriores, ${ }^{1}$ la resiliencia se ocupó de la ciudad y del ámbito digital, posicionándose como una práctica nacida desde el instinto mismo de supervivencia. Esa resiliencia se conformó individualmente, pero en un proceso de conciencia colectiva que, cual unidad celular, terminó manifestándose en colectividad y generando sistemas más complejos que disiparon a los primigenios (Maturana y Varela, 2003: 63).

La organización ciudadana comenzó a generar elaborados organismos de innovación social, expresados en grupos que terminarían por empoderar las acciones solidarias. Un fenómeno inédito de gobernanza² ciudadana se precipitó para asombro de los negacionistas, las autoridades capitalinas y las federales. La ciudadanía estaba en el poder, y todo ese potencial se fundamentaba en la empatía y la agilidad de la información que de a poco comenzaba a regularse.

El miedo dejó paso a la solidaridad y al desapego, las barreras se derrumbaron. Estremecida, la ciudadanía agitaba las calles con la ayuda que podía mover y, sobre todo, en cuanto lo podía hacer. Se improvisaron centros de acopio en plazas, escuelas y diferentes espacios públicos y privados. Espontáneamente, la ayuda material y humana se concentró en torno a la zona centro de la ciudad, donde se acumula más riqueza y un alto uso de los medios digitales. Las colonias Roma, Condesa, Narvarte, Del Valle, concertaban infinidad de acciones, en una suerte de diagrama radial, también directamente relacionado con la cantidad de emergencias en esas zonas. A diferencia de 1985, donde el sismo golpeó a clases socioeconómicas más bajas, muchos llamaron a este "el sismo de la clase media".

\section{Transformación digital}

Para los integrantes de CoDeck, cuya misión está íntimamente relacionada a la transformación digital más allá de la mera cultura digital, ${ }^{3}$ desde las primeras horas se detectaron dos tareas urgentes imbricadas entre sí: 1) moderar la conversación digital estudiando el comportamiento compulsivo y los patrones de uso indiscriminado que encontrábamos en Whatsapp, Twitter y Facebook. Para redirigirlos en función del siguiente y más importante objetivo, 2) la optimización de la ayuda ciudadana.

Nos dimos cuenta que si se estructuraban y digitalizaban los flujos de oferta y demanda para la ayuda a los damnificados, conseguiríamos un conjunto de buenas prácticas que, estandarizadas en los centros de acopio, lograría una verdadera eficiencia en la entrega de los recursos, evitando así el movimiento de cientos de kilos de ayuda y decenas de personas de un lugar al otro, sin conocer la verdadera necesidad y su potencial efectividad. 


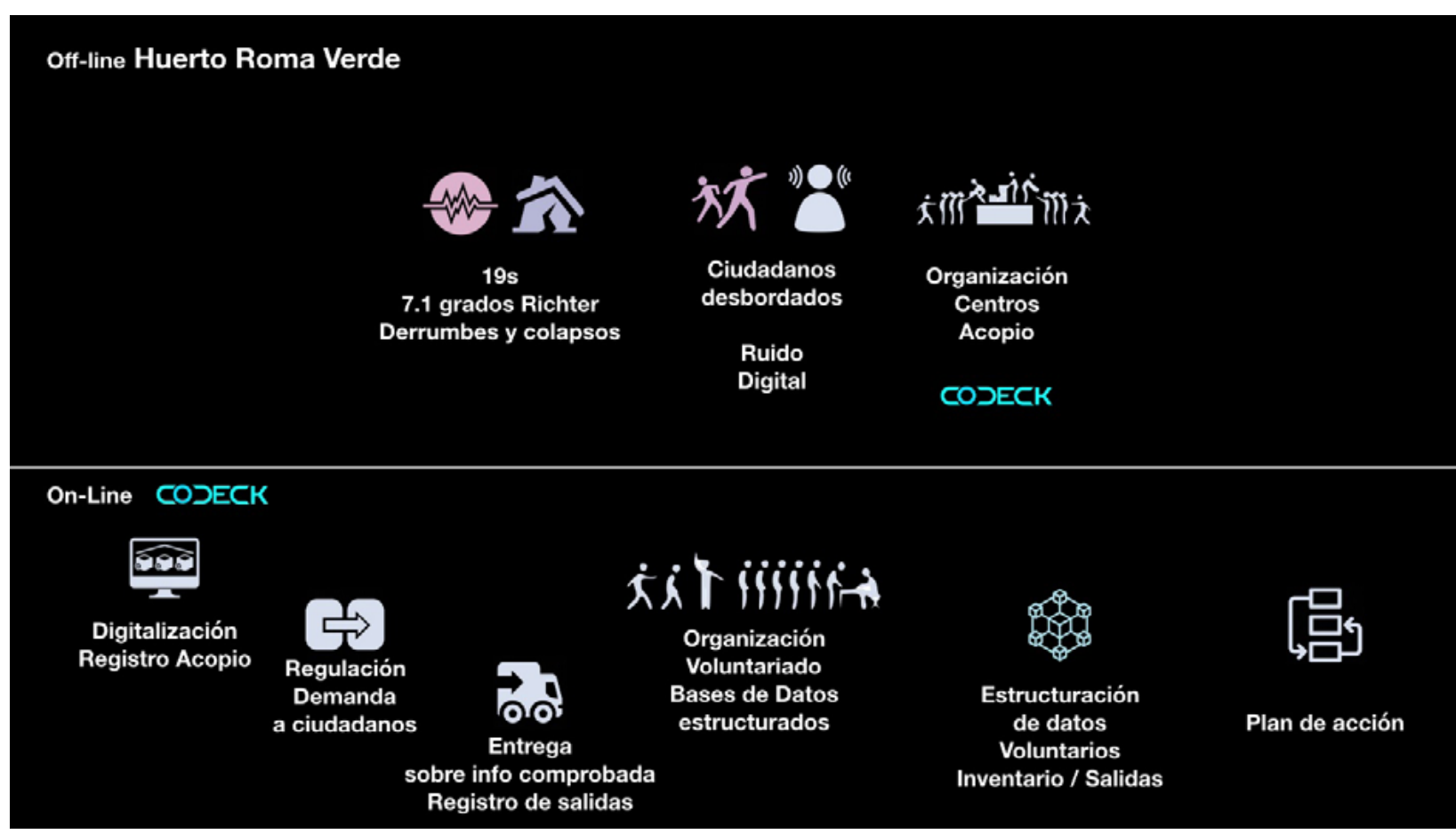

Figura 1. Ruido digital ante el terremoto del $19 s$ y propuesta para la optimización de ayuda ciudadana.
Paradójicamente, esta visión de optimización de los procesos de ayuda sobrepasaba nuestras capacidades desde la iniciativa privada. Tras una rápida exploración del panorama decidimos concentrar nuestros esfuerzos en un solo lugar: el Huerto Roma Verde (HRV). Es por ello que nuestra reflexión gira en torno a la crónica de lo sucedido en ese centro comunitario, sin el deseo de menospreciar otros esfuerzos o de sobre valorar las acciones que se tomaron en el lugar. Sería motivo de un trabajo mucho más extenso el analizar el contexto general de todo lo sucedido en la ciudad durante los sismos de 2017, por ello, estas reflexiones se realizan desde esa delimitación.

Las habilidades de los que nos acercamos al Huerto Roma Verde crearon una atmósfera inédita de innovación social sustentada en la resiliencia y en la confianza mutua, en la disposición para colaborar sin resistencias ni cuestionamientos y en la superposición de un objetivo que transcendía por mucho la individualidad. Esto permitió la coordinación de cientos (con los días serían miles) de personas que trabajaban incansablemente para ayudar a sus semejantes. Con una masa crítica de tales características y en ese contexto extraordinario, comenzaron a alinearse las esferas online y offline, y en menos de 72 horas se estandarizaron los flujos de procesos y se eficientó el trabajo de todos los voluntarios a través de una acción de transformación digital.

\section{Optimización de la ayuda ciudadana}

El volumen de ayuda desmedido que llegaba al HRV aceleró la creación, e iteración, de un sistema de inventario físico que luego se capturaba con rigor en bases de datos. De esta manera, se modelaba la oferta de ayuda al tiempo que se 
4 Se entrevistaron centros de acopio como: Parque México, Explanada Benito Juárez, Cibeles y Lago Tanganica. Vale la pena hacer mención de la

organización CADENA, pues fue la única que contaba con métodos predefinidos y un modelo de acción que sí consideraba la digitalización. Paradójicamente, la velocidad de operación que le proporcionaban estos métodos hicieron irrelevante la colaboración con otros centros de acopio en el ámbito digital. conocía con precisión las carencias de inventario para modelar la demanda, con el fin de satisfacer las carencias en otros puntos de ayuda, zonas de desastres, albergues y comedores comunitarios.

Los inventarios digitalizados, nos permitieron modelar nuestra comunicación con la ciudadanía, pues en pocas palabras: sabíamos que pedir, que se necesitaba realmente y dónde. Este último aspecto se comunicaba con una enorme agilidad a través de medios digitales, pero también gracias a la comunicación verbal contenida en llamadas telefónicas, de modo que el rango de acción pronto se volvió interestatal.

Fue así que comenzamos un mapeo imprescindible de los damnificados y voluntarios con los que podíamos colaborar, utilizando la información que llegaba a través de los medios digitales pero, sobre todo, clasificándola a través de comprobaciones que hacíamos en las llamadas telefónicas e imágenes que nos dieran veracidad acerca de las solicitudes de apoyo. Sobre las mesas aparecían listas escritas a mano, que se convertían en directorios de referencia, cuando estos documentos se intentaban mejorar y pasar en limpio — digitalizar-, el uso demandaba volver a escribir a mano sobre ellos. De forma tal que la innovación social se promovía en cada diálogo de colaboración entre las personas y superaba la intención de digitalizar absolutamente todo, como una manifestación de la cultura digital.

Después de algunos intentos sin éxito de mejorar nuestro sistema de inventario y bases de datos, nos dimos cuenta que al menos una de nuestras metas, en el contexto del Huerto, estaba cubierta, pues la intención primigenia era hallar un modelo accionable que pudiera coordinar los esfuerzos ciudadanos en una base de datos centralizada. Viendo que la metodología ya funcionaba en el Huerto, comenzamos a intentar exportar esos estándares de inventario hacia otros centros de acopio.

En conversaciones telefónicas y encuentros presenciales todo parecía encajar, casi todos los centros que entrevistamos habían implementado lógicas similares en la parte de comprobación de la información e, incluso, intentos de digitalización. ${ }^{4}$ Sin embargo, fue imposible replicar lo que hacíamos en el HRV, pues la sombra de la todavía incipiente cultura digital en la CDMX, y en general del país, nubló nuestras posibilidades. Aunado a esto, hubo muchos elementos que se convirtieron en las barreras más importantes de adopción de las prácticas utilizadas en el Huerto, como son: las condiciones de organización y seguridad de otros centros de acopio, la consideración superficial sobre las prácticas que nosotros sugeríamos al calor del trabajo de acopio y entrega, la falta de visión de algunos líderes de opinión y corporaciones digitales que prefirieron apoyar esfuerzos más cercanos a una cultura digital y no de transformación digital. 

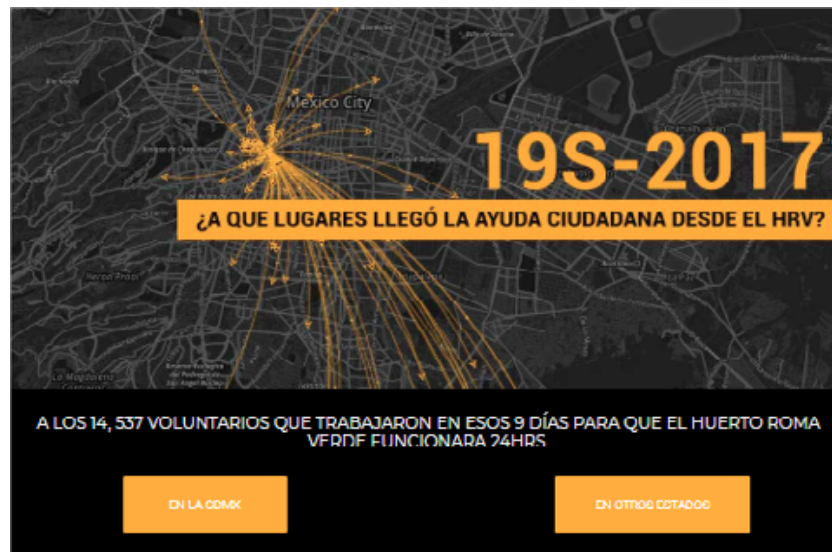

VFRMF FINCIONARA 24HD

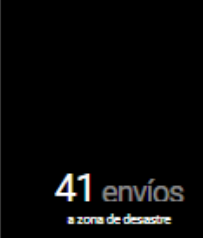

+10 envíos

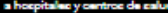

150 brigadas

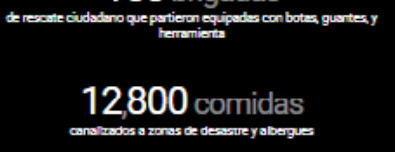

12,800 comidas

\section{7 envíos}

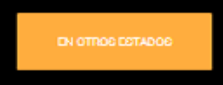

7 ESTADOS / 82 MUNICIPIOS Y DELEGACIONES

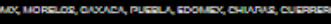

Figura 2. Transparencia de las entregas del HRV.

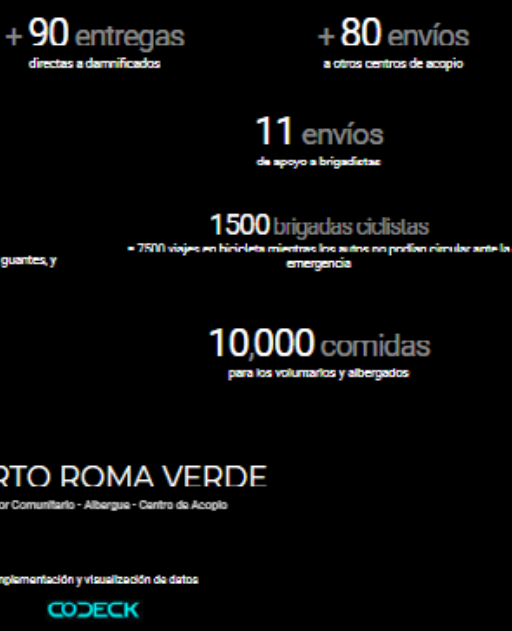

Desde un inicio nos acompañaba y fortalecía la visión de poder dar cuenta a la ciudadanía (que donaba aquellos recursos masiva y desinteresadamente) de los procesos de acopio y entrega que sucedían en y desde el HRV. Se trataba de implementar uno de los beneficios característicos de la transformación digital: la transparencia. Pero esa recompensa no era visible en la inmediatez, no era palpable como otros esfuerzos que sí contribuían en el uso de lo digital, pero sin una fuerza transformadora. A fin de cuentas, el resultado de las buenas prácticas que se aplicaron el Huerto, hoy se pueden ponderar en la visualización de las entregas del HRV.

\section{Moderación de la conversación}

Llevar un control del inventario nos permitía regular la conversación digital del HRV en sus redes sociales, es decir, se intentaba que las donaciones se abocaran a lo verdaderamente necesario mediante la emisión de mensajes en Twitter, Facebook y WhatsApp, principalmente. 
Figura 3. En esta imagen se representa la nube de interacciones con la que se relacionó el HRV en esos días. La falta de adopción de las prácticas dificulta el análisis y segmentación de dichos mensajes.

5 En CoDeck usamos ese término con la intención de identificar audiencias que se conectan a través del contenido, pero que no pueden establecerse como comunidades pues la esencia misma de las plataformas digitales o las llamadas redes sociales, hace que estas agrupaciones sean dinámicas y cambiantes en dependencia de las interacciones, de ahí el símil con las tribus, pues su organización no está delimitada por acuerdos

fijos que propicien su evolución.

6 La Revolución Horizontal Gonzalo Alonso y Alberto Arébalos. cap. La Nueva Modalidad del mensaje.
Intentábamos estandarizar ciertos hashtags y cuentas para organizar la conversación, sin embargo, la inercia en las formas de uso de estas plataformas (como rasgo definitorio del nivel de cultura digital) se erigieron como barreras de adopción. El uso tan personal que cada quien hacía de los mensajes que contenían las necesidades, aunado a la omisión del hashtag, hizo muy complejo el rastreo de los mensajes y la organización de la información en torno al HRV. Entre las prácticas deficientes provocadas por esa "cultura digital" o inercia en las formas de uso, se destacan las siguientes:

- Muchos hacían videos pidiendo lo que se necesitaba en ese momento, sin usar los hashtags o las cuentas sugeridas.

- Otros pedían lo que se necesitaba, pero usaban imágenes creadas por ellos mismos o fotos de las cartulinas donde informábamos las necesidades para y en el contexto offline del HRV, de modo que al no contar con el hashtag o ser simplemente imágenes, tampoco los podíamos rastrear.

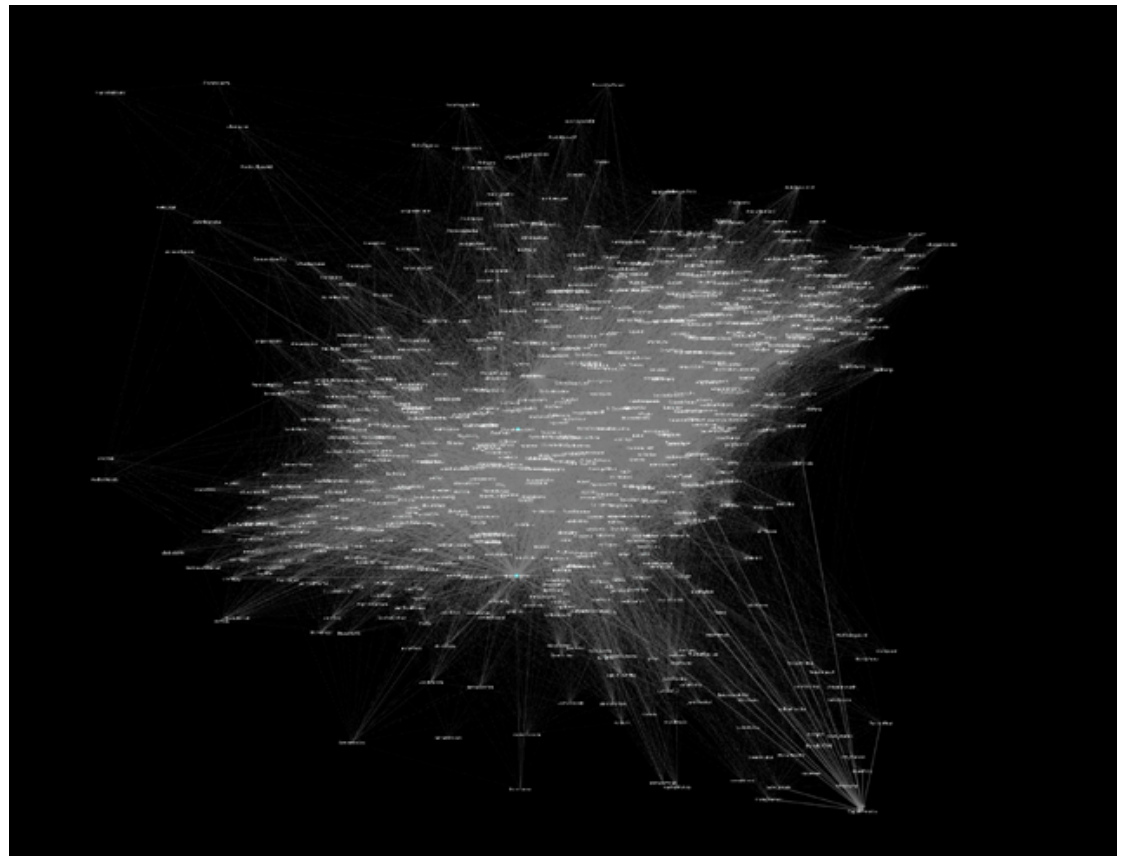

Intentábamos aprovechar la interacción entre el mundo físico offline y el digital online haciendo extensivos a los usuarios presentes en el Huerto Roma Verde tanto las necesidades como las buenas prácticas de los hashtags y las cuentas, con el objetivo de que estos voluntarios los replicaran en sus propias redes, de manera que todas las tribus digitales ${ }^{5}$ que se encontraban inmersas en la ayuda, participaran en la conversación digital. Mientras los medios de comunicación oficiales quedaban fuera de la misma, la ciudadanía no solo ejercía la gobernanza antes mencionada, sino que además moderaba su conversación y, por ende, su logística, bajo sus propias reglas. ${ }^{6}$ Es por ello que la escucha de esta a conversación digital, se hacía fundamental para potenciar la eficiencia de la ayuda ciudadana.

Este intento por sistematizar la conversación le pareció irrelevante a los usuarios al calor de los hechos, evidenciando lo lejos que estamos, como 
Figura 4. Nube de palabras del timeline de Facebook del HRV.
7 INFOTEC Centro de Investigación e Innovación en Tecnologías de la Información

y Comunicación (https:// www.infotec.mx) pertenece a la red de Centros Públicos de Investigación del Consejo

Nacional de Ciencia y

Tecnología (CONACYT) y colaboró en particular con el laboratorio INGEOTEC (http:// www.ingeotec.mx) un equipo de procesamiento de lenguaje natural (NLP) dirigido por el Dr. Elio Villaseñor.

AlApplied (https:// ai-applied.nl) es una empresa holandesa de inteligencia artificial también orientada a la procesamiento de lenguaje natural , con especialidad en redes sociales y medios digitales.

8 Son programas que aprenden a ejecutar una cierta tarea y que mejoran en la ejecución de esa tarea conforme se les entrena.

9 Era importante agregar una categoría de No relacionado (con el 19s) para poder discernir tweets que se publicaban en esos días con otros temas y objetivos

Figura 5. Grupo de tweets procesados y las distintas categorías en las que fueron clasificados. sociedad, de comprender la necesidad de una verdadera cultura digital que nos permita transitar hacia una transformación digital, para no regodearnos en "soluciones" que solo abundan en un contexto de propuesta digital.

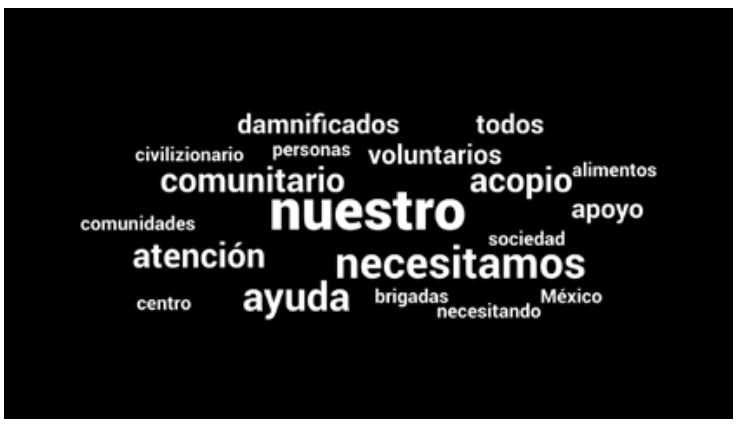

Con el fin de profundizar en la moderación de la conversación digital, y con la ayuda de Emmanuel Flores (del equipo de CoDeck), se movilizó y coordinó la ayuda de una empresa holandesa y un equipo de procesamiento de lenguaje natural en Aguascalientes, para establecer un sistema de escucha de Twiter en tiempo real. Así, nació la alianza AiApllied/Infotec/CoDeckํ quienes nos dimos a la tarea de generar un modelo de clasificación del torrente de tweets en diferentes categorías. Estos modelos trabajan con algoritmos de aprendizaje automatizado (Machine learning en inglés) ${ }^{8}$ a fin de separar en conjuntos todo el torrente de tweets

A grandes rasgos el modelo funciona de la siguiente manera:

1. Definición de siete categorías relevantes dentro de este contexto: Pide apoyo, Información general, Ofrece apoyo, Opinión, Buenos deseos o empatía, Acopio, No relacionado. ${ }^{9}$

2. Generación programas para leer los tweets y clasificarlos.
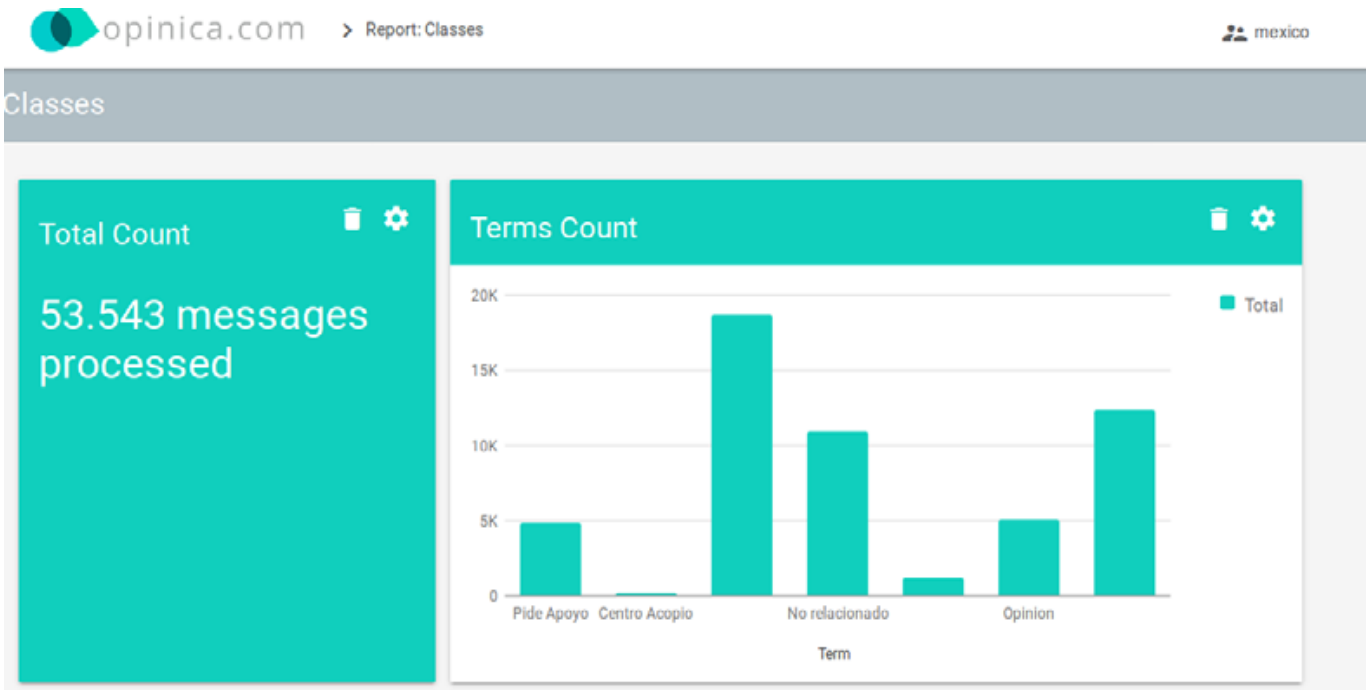
Figuras 6 y 7. De la nube de interacciones del HRV se definen las comunidades y los actores que más propagación generaron.
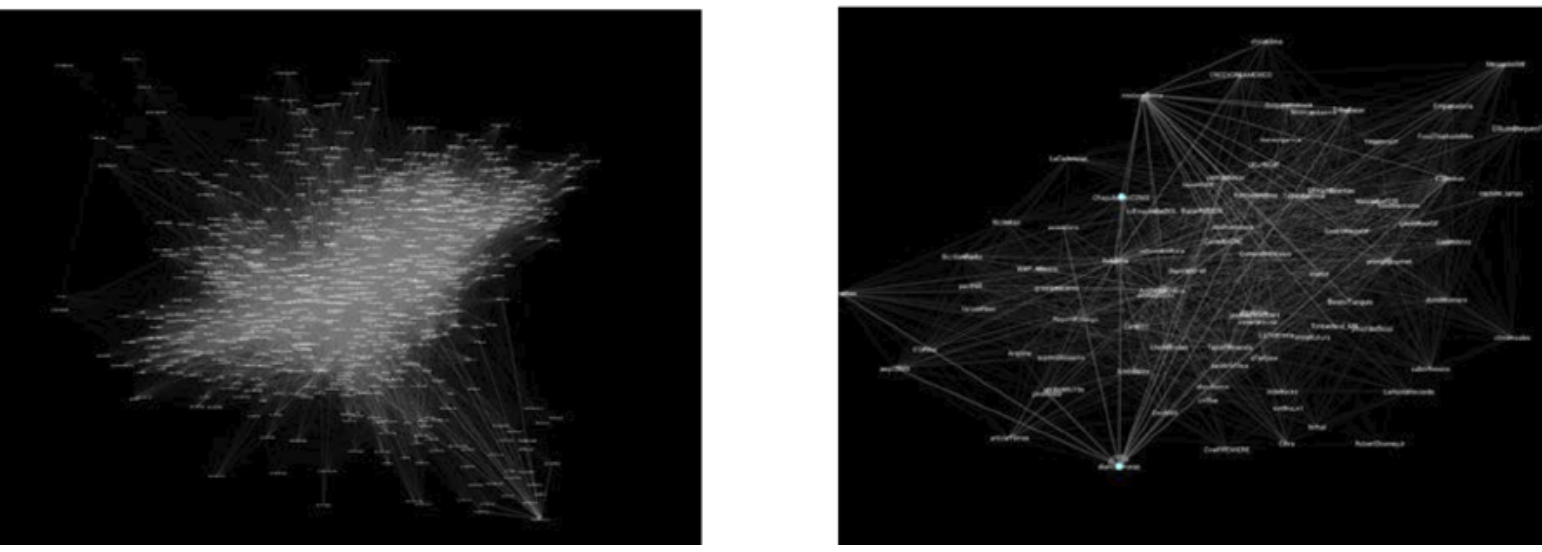

Tras clasificar en conjuntos los mensajes, el modelo de CoDeck se orientaba a los siguientes objetivos: 1) agrupar audiencias por sentimiento, ubicación y el uso del lenguaje; 2) construir audiencias similares basadas en segmentos pequeños a fin de hallar una colección de mensajes más efectivos para esas audiencias; 3) acelerar la distribución masiva de mensajes identificando a influencers o personas que conectaban nuevas comunidades.

10 Este tipo de métodos permite preveer la demanda de un bien, de un servicio o de muchos bienes y servicios en el tiempo, se estudia con funciones matemáticas el comportamiento de

la demanda en un cierto periodo pasado para proyectar a tiempos futuros.

11 Estos modelos son ideales para modelar el comportamiento de individuos (los voluntarios) en un contexto (la ayuda, las tareas). Estas técnicas se basan en la simulación computacional de los

fenómenos, reporducir el fenómeno artificialmente en una o muchas computadoras para estudiarlo y entender que soluciones son mejores.

12 El problema del agente viajero es un problema clásico de los modelos de optimización en transporte (análisis de redes). El planteamiento general es el de un agente que debe recorrer un cierto número de ciudades con el menor esfuerzo (tiempo o dinero) posible.

\section{Conclusiones y aprendizajes}

Los modelos matemáticos son herramientas que permiten aproximarnos a fenómenos de la realidad para hallar soluciones a problemas que pueden ser más o menos complejos. Estos modelos muchas veces requieren información para arribar a conclusiones contundentes.

En este caso, la sociedad organizada conformó la fuente de información de la que se alimentaran distintos modelos. Sin embargo, la estandarización hubiera permitido atender mejor las necesidades durante la emergencia, maximizando el efecto de la ayuda ciudadana, la organización de voluntarios y la clasificación de horarios y habilidades disponibles, para luego poder cruzar los datos con las necesidades y generar un sistema que asignara a cada quién, según sus tiempos y destrezas, ciertas tareas en la ayuda. Y, lo más importante en nuestra visión: minimizar los esfuerzos de la distribución.

Destacamos aquí tres diferentes aplicaciones cuya implementación hubiera sido posible de haber tenido un inventario centralizado:

I. Modelos predictivos de demanda ${ }^{10}$ de bienes y servicios que se alimentaran de las actualizaciones de los informantes en campo.

II. Modelos de agentes autónomos ${ }^{11}$ para optimizar las tareas y tiempos de los voluntarios.

III. Modelos tipo el agente viajero ${ }^{12}$ para la optimización de las rutas de distribución de la ayuda.

Al tener el inventario común y centralizado en una única base de datos, se podrían modelar las necesidades de cada centro de acopio para su comunicación. Pero 
más importante que eso, es la capacidad de aplicar los modelos y algoritmos para hacer más eficaz la recogida de la ayuda en centros de acopio y, a su vez, modelar rutas eficientes de entrega, lo cual significaría más ayuda en menos tiempo y sobre todo ayuda efectiva.

En el HRV teníamos un listado digitalizado de los transportes ciudadanos (contrastados con identificación oficial y placas) a los cuales asignábamos las salidas. Un sistema central que conociera las necesidades de los diferentes tipos de puntos de emergencia, con la recogida de ayuda material y reclutamiento humano previamente digitalizado (en el huerto se registraron más de 4000 personas dispuestas a ser voluntarias), podría sugerir rutas eficientes para la coordinación de los diferentes centros de acopio y sus entregas.

Podemos concluir que el sistema de inventarios, el cual se regía por definiciones genéricas de las cantidades más que por un conteo preciso de la ayuda (las categorías eran: nada, poco, suficiente, sobra.), fue un éxito en el contexto del Huerto Roma Verde tanto para la gestión de los recursos, como para la comunicación de necesidades, así como en el intercambio de material con otros centros de acopio. Y en su aplicación a posteriori fue la base para la generación de la visualización de datos que informa a la población sobre el destino de sus donaciones, erigiéndose en una herramienta de transparencia y confianza entre la ciudadanía (ver la página del Huerto). Sin embargo, la adopción de las buenas prácticas en la emisión de los mensajes para modelar oferta y demanda, se vio coartada por el estado de la cultura digital y algunos factores probablemente emocionales, así como el ruido digital que creaban los patrones de uso indiscriminado de las plataformas digitales.

Un modelo inteligente como el propuesto, puede determinar: qué mensajes difundir, en qué grupos, qué mensajes son vigentes, qué mensajes negativos y cuáles son falsos, incluso qué mensajes apagar o bloquear, pero los cuestionamientos éticos aparecen de inmediato: ¿La magnitud de un desastre como el 19s justifica que cada mensaje compartido se exponga al riguroso escrutinio de la inteligencia artificial y establecer algún tipo de regulación o incluso censura de los mensajes?

Usamos los medios digitales sin una conciencia clara del potencial transformador que podemos implementar con ellos, en ocasiones son medios de comunicación o de expresión que, con el uso desmedido, devienen en un fin en sí mismo en vez de usarlos como medios o herramientas. Sin embargo, el fenómeno de verificación y de cuestionamiento de las fuentes se esparció en una magnitud que podría ser objeto de estudio en este contexto.

Nos queda como especie un largo recorrido para explotar el potencial de las herramientas digitales, y este aprendizaje puede ser violentado por la irrupción de la tecnología en nuestras vidas en muy pocos años, mientras tanto, no parece estar en la agenda de las políticas públicas la preparación para tal transformación digital. 
Figura 8. Propuesta de flujo para eficientar la ayuda.
Los movimientos sociales que se potencian por vías digitales, parecen tener una tendencia efímera como muchas otras cosas en lo digital y no se halla una consecuencia o un reflejo a largo plazo generalizado tras la efervescencia.

Aprendimos el potencial organizativo que podemos alcanzar como ciudadanía, fuimos altamente resilientes ante el trauma y superamos a las instituciones, mostrando un nuevo camino de colaboración sobre el cual son necesarios acuerdos entre gobierno y ciudadanía, pero este sismo no hizo sino poner en evidencia; la desconfianza mutua entre gobernantes y gobernados, la poca capacidad y quizás nula voluntad para integrarse a los procesos de conversación social digital de las agencias de gobierno y la imperiosa necesidad de pertenencia y generación de comunidad que tienen los habitantes de la cDMx.

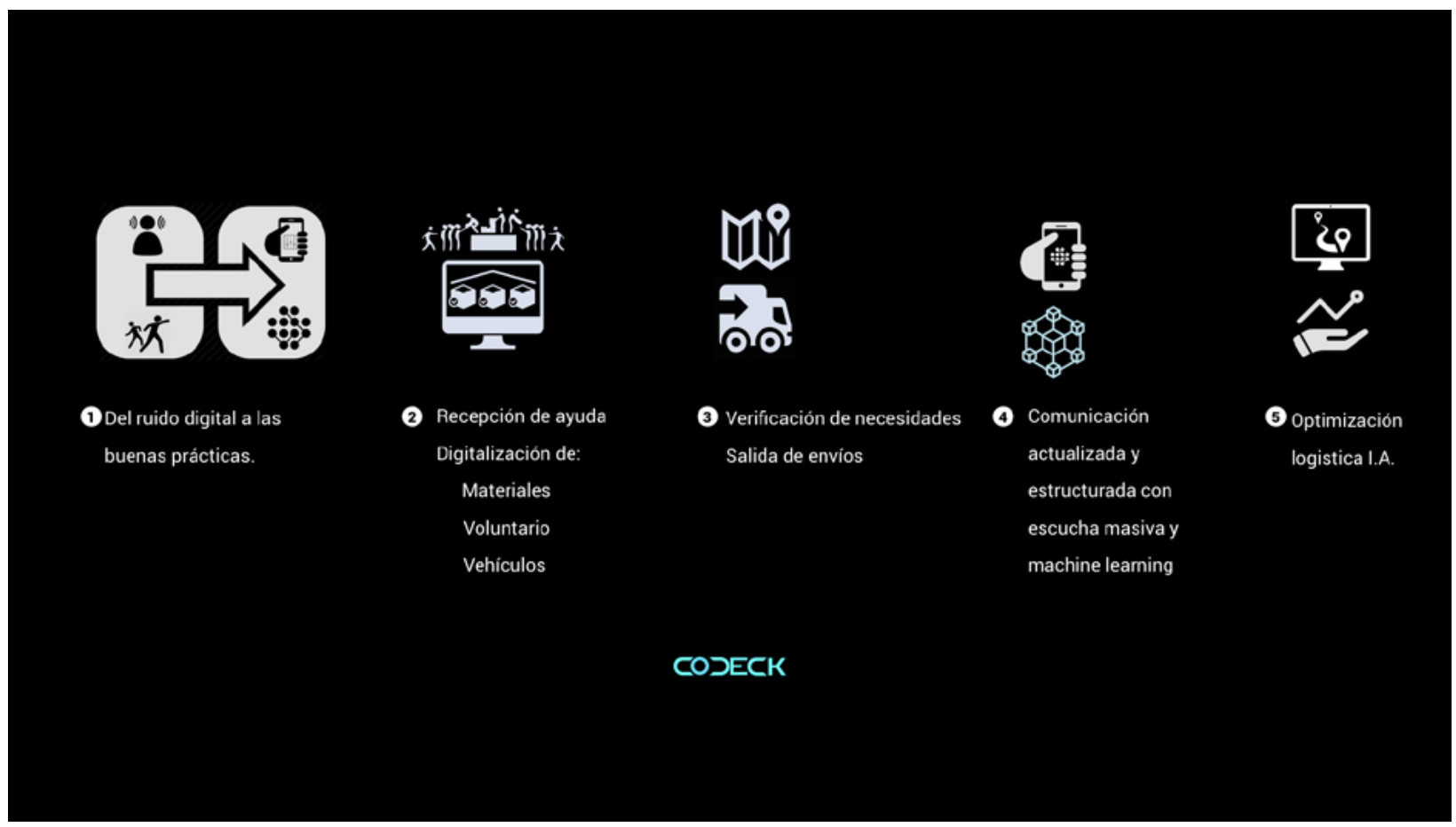

\section{Referencias}

* Charney D. (2004). Psychobiological mechanisms of resilience and vulnerability: implicationsfor successfuladaptation to extremestress. AmJ Psychiatry.161:195-216.

* Chiang, C.L, (2003) Statistical methods of analysis, World Scientific. ISBN981-238310-7 - page 274 section 9.7.4 "interpolation vs extrapolation"

Crooks, Andrew y Heppenstall, A. J. (2012). Introduction to Agent-Based Modelling. DOI: 10.1007/978-90-481-8927-4_5.

- Gobernanza (s.f.). Temas mundiales. Naciones Unidas. Recuperado de http://www. un.org/es/globalissues/governancel. 
* Weisstein, Eric W. (s.f.). Traveling Salesman Problem. Wolfram Research. Recuperado de http://mathworld.wolfram.com/TravelingSalesmanProblem.html

* Problema del viajante (s.f.). En Wikipedia. Recuperado de https://es.wikipedia.org/ wiki/Problema del viajante

- Humberto Maturana y Francisco Varela (2003). De máquinas y seres vivos: autopoieis, la organización de lo vivo. Ed Universitaria Lumen 6ta edición.

- Lawler, E., Lenstra, J., Rinnooy Kan, A. y Shmoys, D. (1985). The Traveling Salesman Problem: A Guided Tour of Combinatorial Optimization. Nueva York: Wiley.

* Sameera Abar, Georgios K., Theodoropoulos, Pierre Lemarinier, Gregory M. P. O'Hare (2017). Agent Based Modelling and Simulation tools: A review of the stateof-art software, Computer Science Review. Vol. 24. pp. 13-33.

* Sean Coughlan (12 de enero de 2017). Qué es la "posverdad", el concepto que puso de moda el "estilo Trump" en Estados Unidos. BBC Mundo.

Shumway, R. H. y Stoffer, D. S. (2000). Time Series Analysis and Its Applications, with R Examples, 2nd ed. Nueva York: Springer-Verlag.

* Waegeman, Willem, De Baets, Bernard y Boullart, Luc (2008). "ROC analysis in ordinal regression learning". Pattern Recognition Letters. 29: 1-9. DOI: 10.1016/j. patrec.2007.07.019.

\section{Cómo citar este artículo}

* Jiménez, Igor y Valdés, Emiliano (2019). Transformación digital en el sismo del 19 de septiembre. Revista Digital Universitaria (RDU). Vol. 20, núm. 2. Dol: http://doi. org/10.22201/codeic.16076079e.2019.v20n2.a5 